\title{
Hysteresis and Fréedericksz thresholds for twisted states in chiral nematic liquid crystals: Minimum-energy path approach
}

\author{
Semen S. Tenishchev, ${ }^{1,2, *}$ Ivan M. Tambovtcev, ${ }^{2, \dagger}$ Alexei D. Kiselev, ${ }^{1,2, \dagger}$ and Valery M. Uzdin ${ }^{2,1}$, $\$$ \\ ${ }^{1}$ ITMO University, Kronverkskiy, 49, 197101, St. Petersburg, Russia \\ ${ }^{2}$ St. Petersburg State University, 199034, St. Petersburg, Russia
}

\begin{abstract}
We study minimum-energy pathways (MEPs) between the branches of metastable helical structures in chiral nematic liquid crystals (CNLCs) subjected to the electric field applied across the cell. By performing stability analysis we have found that, for the branches with non-vanishing half-turn number, the threshold (critical) voltage of the Fréedericksz transition is an increasing function of the free twisting wave number. The curves for the threshold voltage depend on the elastic anisotropy and determine the zero-field critical free twisting number where the director out-of-plane fluctuations destabilize the CNLC helix. For each MEP passing through a first order saddle point we have computed the energy barrier as the energy difference between the saddle-point and the initial structures at different values of the applied field. In our calculations, where the initial approximation for a MEP at the next step was determined by the MEP obtained at the previous step, the electric field dependence of the energy barrier is found to exhibit the hysteresis. This is the hysteresis of electrically driven transition of the saddle-point configuration between the planar and the tilted structures involving out-of-plane director deformations. It turned out that, by contrast to the second-order Fréedericksz transition, this transition is first order and we have studied how it depends on the zenithal anchoring energy strength.
\end{abstract}

\section{INTRODUCTION}

Self-organized soft helical superstructures appear as a manifestation of the chirality caused by the broken mirror symmetry in certain liquids with long-range orientational order known as liquid crystals (LCs) where the molecules tend to align along a unit vector $\hat{\mathbf{n}}(\mathbf{r})$ called the LC director specifying the locally averaged direction of the LC molecules at a point $\mathbf{r}$ [1, 2]. More specifically, in chiral liquid crystals, the presence of chiral molecules (molecules with no mirror plane) gives rise to equilibrium structures forming helical twisting patterns where the director rotates about a twist (helical) axis.

These supramolecular helical structures are behind a unique combination of photonic properties of chiral nematic liquid crystals also known as the cholesteric liquid crystals (CLCs). Tunability of such structures underlies most of the fascinating device applications of CLCs [3, 4]. So controllable manipulation of the CLC helical superstructures presents a challenging problem which is of vital importance for both fundamental and technological reasons $[5-8]$.

The continuum theory of CLCs is formulated in terms of the Frank-Oseen free energy functional

$$
\begin{aligned}
& F_{\mathrm{el}}[\mathbf{n}]=\int_{V} f_{\mathrm{el}} \mathrm{d} v, \quad f_{\mathrm{el}}=\frac{1}{2}\left\{K_{1}(\nabla \cdot \mathbf{n})^{2}\right. \\
& +K_{2}\left[\mathbf{n} \cdot \nabla \times \mathbf{n}+q_{0}\right]^{2}+K_{3}[\mathbf{n} \times(\nabla \times \mathbf{n})]^{2} \\
& \left.-K_{24} \operatorname{div}(\mathbf{n} \operatorname{div} \mathbf{n}+\mathbf{n} \times(\nabla \times \mathbf{n}))\right\},
\end{aligned}
$$

where $f_{\mathrm{el}}$ is the elastic free energy density; $K_{1}, K_{2}, K_{3}$ and $K_{24}$ are the splay, twist, bend and saddle-splay Frank elastic constants,respectively. The bulk free energy (1) contains a chiral term proportional to the parameter $q_{0}$, the free twist wave number or the free twisting number, giving the pitch $P_{0}=2 \pi /\left|q_{0}\right|$ of equilibrium helical structures in unbounded CLCs.

An efficient method widely used to prepare CLCs is doping nematic LC mixtures with chiral additives that induce a helical structure [2, 8]. For photosensitive chiral dopants (photoswitches), their helical twisting power and thus the CLC equilibrium helix pitch $P_{0}$ can be controlled by light through photoinduced changes in chiral molecular switch conformation that influence the LC's helical twisting power 9 16]. Phototunability of the helix pitch leads to a variety of technologically promising effects such as the phototunable selective reflection, i.e. a light-induced change in the spectral position of the bandgap [17 20].

An important point is that multiple metastable twist states in CLC cells generally appear as a result of competing influences of the bulk and the surface contributions to the free energy leading to frustration [2, 21] and giving rise to multiple local minima of the energy [22]. Properties of the metastable helical structures are determined by the anchoring energy and the free twisting number $q_{0}$. The latter plays the role of the governing parameter for the pitch transitions theoretically studied in Refs. [13, 23 29]. These transitions occur between different branches of metastable states and, in particular, manifest themselves in a jump-like temperature dependence of selective light transmission spectra [30 33.

Geometrically, such transitions are related to the free energy pathways connecting pairs of metastable helical states which are local minima (minimizers) of the free energy landscape viewed as a multidimensional free energy

\footnotetext{
* tenischev.semen@gmail.com

† Email address: imtambovtcev@gmail.com

$\ddagger$ Email address: alexei.d.kiselev@gmail.com

$\S$ Email address: v_uzdin@mail.ru
} 
surface 34. The key elements associated with the transitions are the minimum energy paths (MEPs) between the initial and final states on the free energy surface.

Every point on such a pathway is a free energy minimum in all but a certain direction in the configuration space of CLC director structures. It represents a path with the maximal statistical weight and provides a scenario of the most probable transition between the states.

Maximum along the MEP determines the transition state which is a saddle point on the free energy surface. The energy barrier separating the states can be found as the difference between the saddle point energy and the energy of the initial state. Such energy barriers are used to assess the effect of thermally activated transitions within the framework of the rate theory [35, 36. The barrier heights combined with the Arrhenius formula were also employed to estimate the rate of transitions between metastable twist states in LC cells with strong anchoring conditions [37, 38. The two-dimensional Landau-de Gennes theory was used to study the MEPs of the planar bistable LC device depending on the anchoring conditions 39.

In Ref. 40, 41, the approach based on MEPs was applied to explore field-induced distortions of the free energy landscape in CLC cells subjected to external (magnetic or electric) fields. These distortions are known to lead to a variety of field-induced orientational effects such as the Fréedericksz and unwinding transitions. When the electric field is applied across the CLC cell, these effects crucially depend on a number of factors such as the cell thickness $L$, the pitch $P_{0}$, the applied voltage $U$, the anchoring conditions, elastic and dielectric properties of the CLC material 42 49].

This geometry where the CLC cell with planar anchoring conditions is subjected to the field applied along the twisting axis of the vertically aligned planar helical structure will be our primary concern. In this paper we shall use the geodesic nudged elastic band (GNEB) method 34 to calculate MEPs and the energy barriers for the transitions between metastable twisted states that belong to the branches with different half-turn numbers. As opposed to the case of unwinding transition with negligibly small free twisting number previously studied in Ref. [41, it turned out that the half-turn numbers of metastable helix state branches and the elastic anisotropy are the governing factors for $q_{0}$-dependence of the critical field of the Fréedericksz transition. As a consequence the Fréedericksz thresholds for the helical structures involved in the transitions are generally different and we shall study how the free energy barriers of such transitions depend on the electric field and the anchoring conditions.

The layout of the paper is as follows. General relations that determine the characteristics of the helical structures in CLC cells are given in Sec. II. The the Fréedericksz thresholds are computed by performing stability analysis in Sec. III. Then in Sec. IV we outline the numerical procedure that we use to compute MEPs and the energy barriers. Finally, in Sec. V we discuss our results and make some concluding remarks.

\section{FREE ENERGY}

We consider the planar confining geometry of a CLC cell of thickness $L$, where CLC is placed between two parallel bounding plates: $z=-L / 2$ (lower substrate) and $z=L / 2$ (upper substrate). Anchoring conditions at the substrates are planar with the preferred orientation of molecules at the lower (upper) plate defined by the vector of easy orientation $\hat{\mathbf{e}}_{-}\left(\hat{\mathbf{e}}_{+}\right)$

$$
\hat{\mathbf{e}}_{ \pm}=\cos \psi_{ \pm} \hat{\mathbf{x}}+\sin \psi_{ \pm} \hat{\mathbf{y}}
$$

where a hat will indicate unit vectors.

As in Ref. 41, the anchoring energy $W_{\nu}(\mathbf{n})$ that enters the surface contribution to the CLC free energy functional:

$$
\begin{aligned}
& F[\mathbf{n}, \mathbf{E}]=F_{b}[\mathbf{n}, \mathbf{E}]+F_{s}[\mathbf{n}], \\
& F_{s}[\mathbf{n}]=\sum_{\nu= \pm 1} \int_{z=\nu L / 2} W_{\nu}(\mathbf{n}) \mathrm{d} s
\end{aligned}
$$

where $\mathbf{E}$ is the electric field, will be taken in the form of Rapini-Papoular potential [50]:

$$
\begin{aligned}
& W_{\nu}(\mathbf{n})=\frac{W_{\phi}^{(\nu)}}{2}\left[1-\left(\mathbf{n} \cdot \hat{\mathbf{e}}_{\nu}\right)^{2}\right]_{z=\nu L / 2} \\
& +\left.\frac{W_{\theta}^{(\nu)}-W_{\phi}^{(\nu)}}{2}(\mathbf{n} \cdot \hat{\mathbf{z}})^{2}\right|_{z=\nu L / 2}
\end{aligned}
$$

where $W_{\phi}^{(+)}\left(W_{\phi}^{(-)}\right)$and $W_{\theta}^{(+)}\left(W_{\theta}^{(-)}\right)$are the azimuthal and the polar anchoring strengths at the upper (lower) substrate.

For the CLC director

$$
\mathbf{n} \equiv \mathbf{n}(\theta, \phi)=\cos \theta(\cos \phi \hat{\mathbf{x}}+\sin \phi \hat{\mathbf{y}})+\sin \theta \hat{\mathbf{z}}
$$

parameterized by the tilt and the azimuthal angles, $\theta=$ $\theta(z)$ and $\phi=\phi(z)$, the surface potential (4) assumes the following form:

$$
\begin{aligned}
& F_{s}[\mathbf{n}] / A=\sum_{\nu= \pm 1}\left[\frac{W_{\phi}^{(\nu)}}{2} \cos ^{2} \theta_{\nu} \sin ^{2}\left(\phi_{\nu}-\psi_{\nu}\right)+\right. \\
& \left.\frac{W_{\theta}^{(\nu)}}{2} \sin ^{2} \theta_{\nu}\right]
\end{aligned}
$$

where $A$ is the area of the substrates; $\theta_{\nu} \equiv \theta(\nu L / 2)$ and $\phi_{\nu} \equiv \phi(\nu L / 2)$. The bulk part of the free energy functional (3)

$$
F_{b}[\mathbf{n}, \mathbf{E}]=F_{\mathrm{el}}[\mathbf{n}]+F_{\mathrm{E}}[\mathbf{n}, \mathbf{E}]
$$

is a sum of the elastic energy $F_{\mathrm{el}}[\mathbf{n}]$ given by Eq. (1) and the energy of interaction between the electric field $\mathbf{E}$ and 


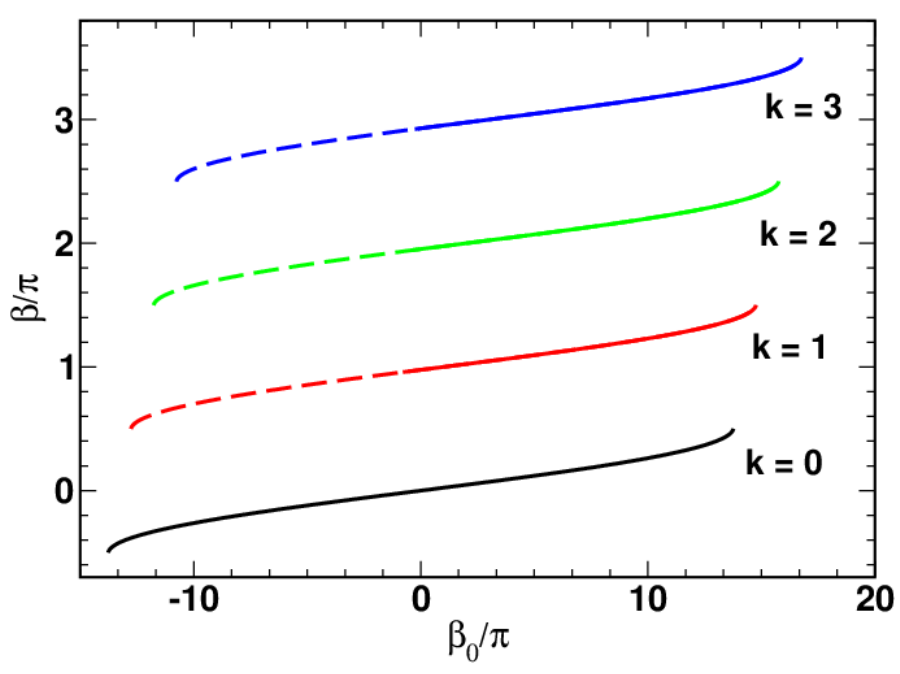

Figure 1: Twist parameter versus the free twisting number $\beta_{0} / \pi=q_{0} L / \pi$ at different values of the half-turn number, $k$. Dashed lines indicate the states unstable with respect to out-of-plane fluctuations.

CLC molecules, $F_{\mathrm{E}}[\mathbf{n}, \mathbf{E}]$. After substituting the CLC director (5), into the elastic energy (1) we have

$$
\begin{aligned}
& F_{\mathrm{el}}[\mathbf{n}] / A=\frac{1}{2} \int_{-L / 2}^{L / 2}\left\{K_{1}(\theta)\left[\theta^{\prime}\right]^{2}+\right. \\
& \left.K_{2}(\theta) \cos ^{2} \theta\left[\phi^{\prime}\right]^{2}-2 C(\theta) \phi^{\prime}+K_{2} q_{0}^{2}\right\} \mathrm{d} z \\
& K_{i}(\theta)=K_{i} \cos ^{2} \theta+K_{3} \sin ^{2} \theta, C(\theta)=q_{0} K_{2} \cos ^{2} \theta
\end{aligned}
$$

where prime stands for derivative with respect to $z$.

We also assume that the electric field is normal to the substrates $\mathbf{E}=E_{z}(z) \hat{\mathbf{z}}$ with $E_{z}(z)=-V^{\prime}(z)$, where $V(z)$ is the electrostatic potential, and the applied voltage, $U=V(-L / 2)-V(L / 2)$, is fixed So, the electrostatic part of the energy

$$
F_{\mathrm{E}}=-\frac{1}{2} \int_{V}(\mathbf{E} \cdot \mathbf{D}) \mathrm{d} v
$$

where $\mathbf{D}=\boldsymbol{\epsilon} \mathbf{E}$ is the electric dispacement and $\boldsymbol{\epsilon}$ is the dielectric tensor, takes the form of nonlocal functional:

$$
F_{\mathrm{E}} / A=-\frac{U^{2}}{2 E[\theta]}, \quad E[\theta]=\int_{-L / 2}^{L / 2} \frac{\mathrm{d} z}{\epsilon_{z z}(\theta)},
$$

where $\epsilon_{z z}(\theta)=\epsilon_{\perp}+\epsilon_{a} \sin ^{2} \theta, \epsilon_{a}=\epsilon_{\|}-\epsilon_{\perp} ; \epsilon_{\perp}$ and $\epsilon_{\|}$are the dielectric constants giving the principal values of $\epsilon$.

In our numerical calculations, we shall use the Frank elastic constants typical for $5 \mathrm{CB}$ [51]: $K_{1}=4.5 \mathrm{pN}, K_{2}=$ $3.0 \mathrm{pN}, K_{3}=6.0 \mathrm{pN}$ and restrict our analysis to the case of the symmetric CLC cell of the thickness $L=5 \mu \mathrm{m}$ with $W_{\phi}^{( \pm)} \equiv W_{\phi}=0.05 \mathrm{~mJ} / \mathrm{m}^{2}$ and $\hat{\mathbf{e}}_{ \pm}=\hat{\mathbf{x}}\left(\psi_{ \pm}=0\right)$.

\section{STABILITY AND FREEDERICKSZ \\ THRESHOLDS OF PLANAR HELICAL STRUCTURES}

In this section we perform stability analysis for the uniform standing helix state with the twist axis normal to the substrates known as the planar Grandjean structure (texture)

$$
\begin{aligned}
& \mathbf{n}_{0} \equiv \mathbf{n}(0, u(z))=\cos u \hat{\mathbf{x}}+\sin u \hat{\mathbf{y}}, \\
& u(z)=q z+\phi_{0},
\end{aligned}
$$

where $q=2 \pi / P$ is the helix wave number and $P$ the helix pitch. To this end, we begin with the expression for the distorted director field

$$
\mathbf{n}(\delta \theta, u(z)+\delta \phi) \approx \mathbf{n}_{0}+\delta \mathbf{n}_{0}, \delta \mathbf{n}_{0}=\delta \phi \mathbf{n}_{1}+\delta \theta \mathbf{n}_{2},
$$

where the angles $\delta \phi$ and $\delta \theta$ describe in-plane and out-ofplane deviations of the director, respectively; the vectors $\mathbf{n}_{1}$ and $\mathbf{n}_{2}$ are

$$
\mathbf{n}_{1}=-\sin u(z) \hat{\mathbf{x}}+\cos u(z) \hat{\mathbf{y}}, \quad \mathbf{n}_{2}=\hat{\mathbf{z}} .
$$

The free energy of the director field (13) can now be expanded up to second order terms in the fluctuation field $\boldsymbol{\psi}=\left(\begin{array}{c}\delta \phi \\ \delta \theta\end{array}\right)$ and its derivatives

$$
\begin{aligned}
& F[\mathbf{n}] \approx F\left[\mathbf{n}_{0}\right]+F^{(2)}[\boldsymbol{\psi}], \\
& F^{(2)}[\boldsymbol{\psi}]=\int_{V} f_{b}^{(2)}[\boldsymbol{\psi}] \mathrm{d} v+\sum_{\nu= \pm 1} \int_{z=\nu L / 2} W_{\nu}^{(2)}(\boldsymbol{\psi}) \mathrm{d} s
\end{aligned}
$$

The second order variation of the free energy $F^{(2)}[\boldsymbol{\psi}]$ is a bilinear functional which represents the energy of the director fluctuations written in the harmonic (Gaussian) approximation.

In what follows we shall restrict our consideration to the case of fluctuations invariant with respect to in-plane translations, so that $\boldsymbol{\psi} \equiv \boldsymbol{\psi}(z)$. In this case the fluctuation energy per unit area is

$2 F^{(2)}[\boldsymbol{\psi}] / A=\int_{-L / 2}^{L / 2} \boldsymbol{\psi}^{\dagger} \hat{K} \boldsymbol{\psi} \mathrm{d} z+\left.\sum_{\nu= \pm 1} \boldsymbol{\psi}^{\dagger} \hat{Q}^{(\nu)} \boldsymbol{\psi}\right|_{z=\nu L / 2}$,

where the expressions for the operators $\hat{K}$ and $\hat{Q}^{(\nu)}$ are given by

$$
\begin{aligned}
\hat{K} & =\left(\begin{array}{cc}
-K_{2} \partial_{z}^{2} & 0 \\
0 & -K_{1} \partial_{z}^{2}+q^{2} K_{q}-\epsilon_{a} E^{2}
\end{array}\right), \\
\hat{Q}^{(\nu)}= & \nu\left(\begin{array}{cc}
K_{2} \partial_{z} & 0 \\
0 & K_{1} \partial_{z}
\end{array}\right) \\
& +\left(\begin{array}{ccc}
W_{\phi}^{(\nu)} \cos 2 u_{\nu} & 0 \\
0 & W_{\theta}^{(\nu)}-W_{\phi}^{(\nu)} \sin ^{2} u_{\nu}
\end{array}\right),
\end{aligned}
$$




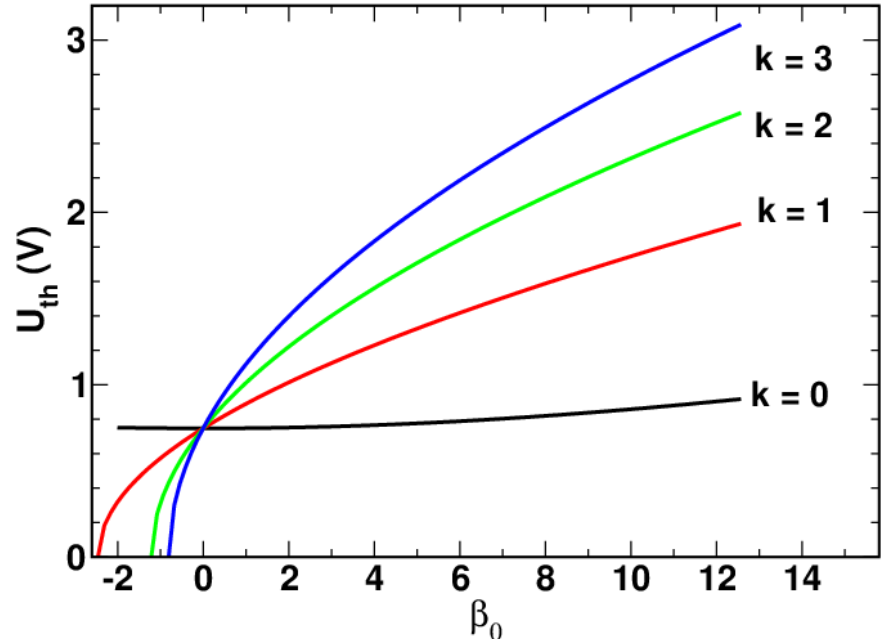

(a) $5 \mathrm{CB}$

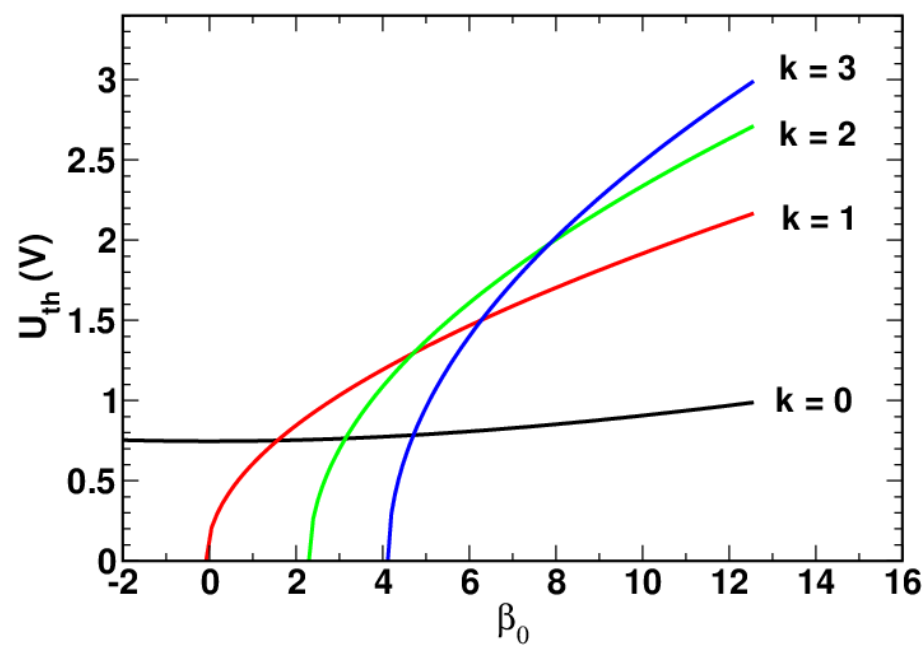

(b) One-constant approximation with $K_{i}=K_{1}$

Figure 2: Critical voltage of the Fréedericksz transition as a function of the free twisting number parameter $\left(\beta_{0}=q_{0} L\right)$ for the branches with the half-turn numbers $0 \leq k \leq 3$. The curves are computed from Eq. (28) for the case of strong polar anchoring.

$E=U / L, u_{\nu}=u(\nu L / 2)-\psi_{\nu}$ and $K_{q}$ is the effective elastic constant

$$
K_{q}=K_{3}-2 K_{2}\left(1-q_{0} / q\right) .
$$

Spectrum of $\hat{K}$ can be computed by solving the boundary-value problem:

$$
\begin{array}{r}
\hat{K} \boldsymbol{\psi}_{\lambda}=\lambda \boldsymbol{\psi}_{\lambda}, \\
\left.\hat{Q}^{(\nu)} \boldsymbol{\psi}_{\lambda}\right|_{z=\nu L / 2}=0,
\end{array}
$$

where $\lambda\left(\psi_{\lambda}\right)$ stands for the eigenvalue (the eigenmode). From Eqs. $\sqrt{18)}-(19)$ the operators $\hat{K}$ and $\hat{Q}^{(\nu)}$ are both diagonal, so that the in-plane and out-of-plane fluctuations can be treated separately. An important point is that the helical configuration 12 is locally stable provided that all the eigenvalues are positive.

For the case of the symmetric CLC cell, stability analysis with respect to in-plane fluctuations performed in Ref. 26 shows that there are different branches of metastable helical states which are labelled by the halfturn number, $k \in \mathbb{N}$, and where the twisting parameter $(k-1 / 2) \pi<q L \equiv \beta<(k+1 / 2) \pi$ is related to the free twisting number, $q_{0}$, as follows

$$
q_{0} L \equiv \beta_{0}=\beta+(-1)^{k} w_{\phi} \sin \beta, \quad w_{\phi} \equiv \frac{W_{\phi} L}{2 K_{2}} .
$$

The branches with $0 \leq k \leq 3$ and $w_{\phi} \approx 41.67\left(W_{\phi}=\right.$ $0.05 \mathrm{~mJ} / \mathrm{m}^{2}$ ) are depicted in Fig. 1 .

We now study stability of the helical structures with respect to the out-of-plane fluctuations. To this end we replace $\lambda$ with $K_{1}(2 / L)^{2} \lambda$ and rewrite the eigenvalue prob- lem $21-22$ for $\theta$ in the following form:

$$
\begin{aligned}
& {\left[\partial_{\tau}^{2}+\left(-r_{q}+\epsilon_{a} U^{2} / K_{1}\right) / 4+\lambda\right] \theta_{\lambda}(\tau)=0,} \\
& {\left[ \pm \partial_{\tau} \theta_{\lambda}+w_{\theta}^{( \pm)} \theta_{\lambda}\right]_{\tau= \pm 1}=0,} \\
& r_{q}=(q L)^{2} K_{q} / K_{1}=\left(r_{3}-2 r_{2}\right) \beta^{2}+2 r_{2} \beta_{0} \beta, \\
& w_{\theta}^{(\nu)} \equiv \frac{\left(W_{\theta}^{(\nu)}-W_{\phi}^{(\nu)} \sin ^{2} u_{\nu}\right) L}{2 K_{1}}, \beta=q L,
\end{aligned}
$$

where $\tau \equiv 2 z / d, r_{i} \equiv K_{i} / K_{1}$ and $\beta_{0}=q_{0} L$.

The stability condition $\lambda>0$ can now be readily written as follows

$$
U<U_{\mathrm{th}}=\sqrt{\left(4 \lambda_{\min }+r_{q}\right) K_{1} / \epsilon_{a}}
$$

where $\lambda_{\min }$ is the lowest eigenvalue of the problem (24)(25) computed at $r_{q}=0$ and $U=0$. The expression on the right hand side of the inequality (28) gives the critical voltage of the Fréedericksz transition. Above this voltage the applied electric field makes the vertically standing helix unstable with respect to out-of-plane director fluctuations.

When the polar anchoring is strong at both substrates, $W_{\theta}^{( \pm)} \rightarrow \infty$, the eigenvalue $\lambda_{\min }$ is known:

$$
\lambda_{\min }=\left(\kappa_{\min }\right)^{2}=\pi^{2} / 4 .
$$

Otherwise, $\kappa_{\min }$ is below $\pi / 2$ and can be computed as the root of the transcendental equation deduced in Appendix of Ref. 26]

$$
\tan 2 \kappa_{\min }=\frac{\kappa_{\min }\left(w_{\theta}^{(+)}+w_{\theta}^{(-)}\right)}{\kappa_{\min }^{2}-w_{\theta}^{(+)} w_{\theta}^{(-)}},
$$




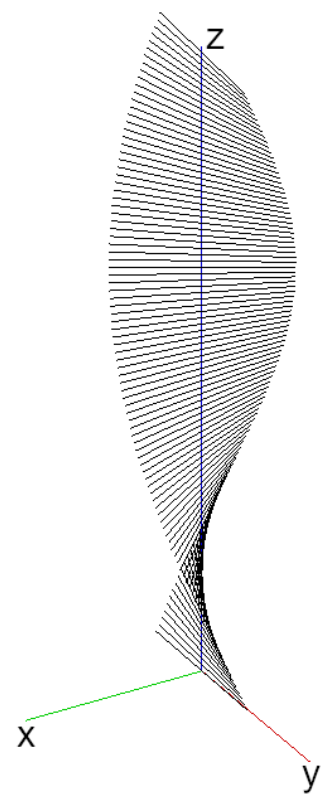

(a) $k=1, U=0 \mathrm{~V}$

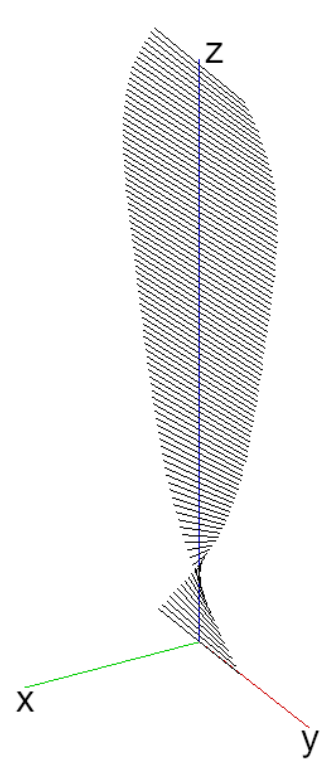

(b) $k=1, U=1.4 \mathrm{~V}$

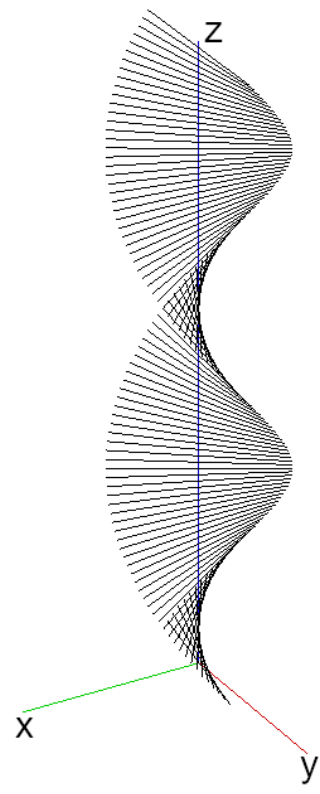

(c) $k=2, U=0 \mathrm{~V}$

Figure 3: Metastable helical configurations: (a) field free one-half-turn structure with $k=1$; (b) distorted one-half-turn structure at $U=1.4 V$; and (c) field free one-full-turn structure with $k=2$. Parameters are:

$$
W_{\phi}^{( \pm)} \equiv W_{\phi}=0.05 \mathrm{~mJ} / \mathrm{m}^{2}, W_{\theta}^{( \pm)} \equiv W_{\theta}=0.5 \mathrm{~mJ} / \mathrm{m}^{2} \text { and } q_{0} L=4.725
$$

where $0<\kappa_{\min } \leq \pi / 2$.

The curves for the critical voltage plotted against the free twisting number are shown in Fig. 2. The results evaluated using the one-constant approximation (see Fig. 2b) are compared with the critical field computed for the elastic constants listed at the end of Sec. II. It can be seen that, at non-vanishing half-turn numbers, in both cases the critical field (Fréedericksz threshold) for each branch grows with the free twisting number $q_{0}$. Given the branch with $k \geq 1$, the value of $q_{0}$ at which the threshold vanishes determines the boundary point giving the lowest free twisting number of locally stable structures. When $q_{0}$ is below this value, the helical states are unstable with respect out-of-plane fluctuations. In Fig. 1 such structures are indicated by the dashed lines. A comparison between the curves presented in Figs. 2a and 2b also shows that the elastic anisotropy has a profound effect on the free twisting number dependence of the Fréedericksz threshold. Our concluding remark in this section is that we have additionally estimated the Fréedericksz thresholds numerically. The numerical results are found to be in excellent agreement with the theoretical predictions.

\section{NUMERICAL RESULTS}

In this section we present the results for the transition between the twisted states with the half-turn numbers $k=1$ (one-half-turn structure) and $k=2$ (one-full-turn structure). The director distributions for these states should be solutions of the stationary point boundary problem (the Euler-Lagrange equations supplemented with the corresponding boundary conditions). In order to find the distributions shown in Fig. 3 we have started from the initial approximation for the director structure and then minimized the energy using the velocity projection algorithm [52. The Fréedericksz thresholds for the helical states with $k=1$ and $k=2$ can be theoretically estimated using formula 28 . So, we have the following estimates: $\left.U_{\mathrm{th}}^{(1)} \equiv U_{\mathrm{th}}\right|_{k=1} \approx 1.3 \mathrm{~V}$ and $\left.U_{\text {th }}^{(2)} \equiv U_{\text {th }}\right|_{k=2} \approx 1.67 \mathrm{~V}$ at $\beta_{0}=4.725$. In particular, as is illustrated in Fig. 3b, the one-half-turn structure becomes distorted when the applied voltage $U=1.4 \mathrm{~V}$ is above $U_{\mathrm{th}}^{(1)}$.

Note that the above states being different in the parity of half turns are topologically distinct. As a result, in the strong anchoring limit, the one-half-turn helix cannot be smoothly deformed into the one-full-turn structure without destroying the local degree of molecular ordering. Contrastingly, in the weak anchoring regime, these states are local minima of the free energy surface separated by the finite energy barriers.

In this section, dependence of the energy barriers on the applied voltage will be the subject of our primary concern. For this purpose, we, following our previous work [41, shall use the minimum-energy path (MEP) approach. Leaving aside technical details about the numerical procedure described in Refs. [40, 41, we just 


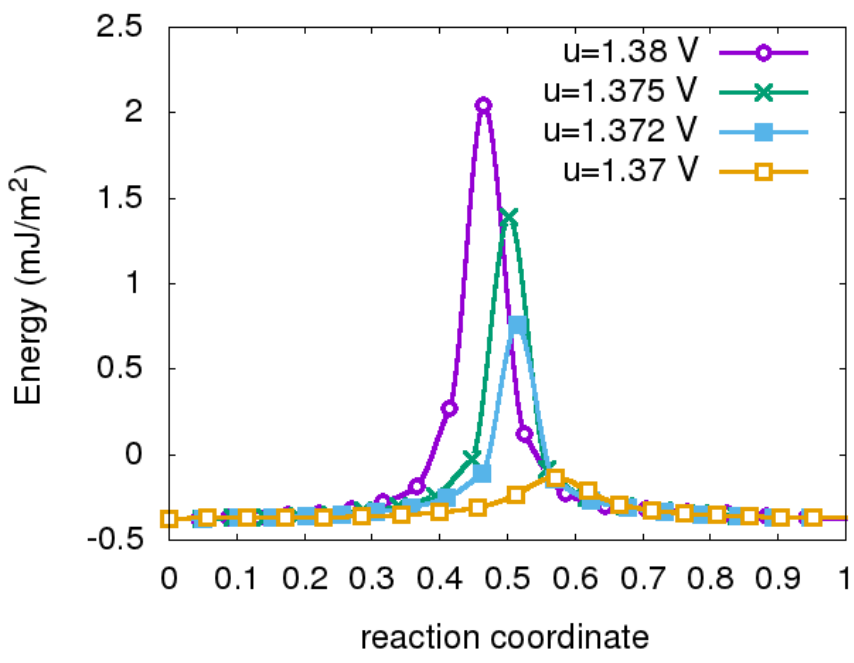

(a) Director slippage-to-anchoring breaking transition.

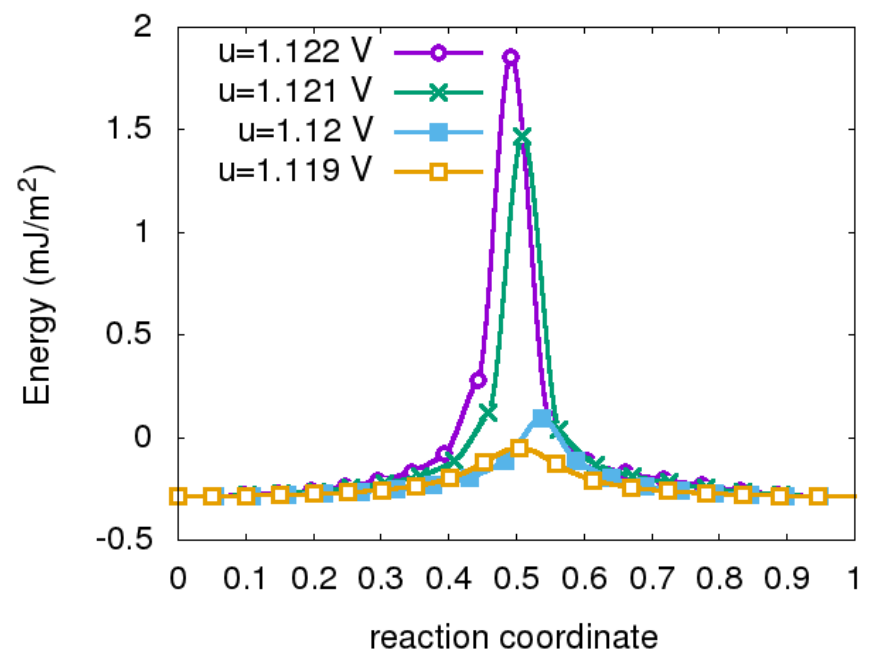

(b) Anchoring breaking-to-director slippage transition.

Figure 4: Energy per unit area along the minimum energy paths at different voltages. Parameters are listed in the caption of Fig. 3.

briefly comment on the geodesic nudged elastic band (GNEB) method to find MEPs between local minima on the curved manifolds 34.

This approach involves taking an initial guess of a path between the two minima of the free energy surface and systematically bringing that to the nearest MEP. A path is represented by a discrete chain of states, or "images", of the system, where the first and the last image are placed at the local energy minima corresponding to the initial and final metastable configurations. In order to distribute the images evenly along the path, springs are introduced between adjacent images. At each image, a local tangent to the path needs to be estimated, and the force guiding the images towards the nearest MEP is defined as the sum of the transverse component of the energy antigradient plus the component of the spring force along the tangent to the path. The position of intermediate images is then adjusted so as to zero the GNEB forces. The position of the maximum (saddle point) along the MEP was found using Climbing Image algorithm [34.

An important point is that the MEP connecting the metastable structures generally depends on the starting approximation for the path. Variations in the initial approximations may produce different MEPs. In Ref. 41, we have used different initial paths to study two scenarios of the unwinding transition: (a) the director slippage transitions where $\theta \approx 0$; and (b) the anchoring breaking transitions that involve tilted CLC states.

In our calculations, similar to the director slippage scenario, the initial (director slippage) approximation for the MEP at the starting value of voltage $U_{0}$ involves the planar $(\theta=0)$ twisted structures of the form (12) assuming that the azimuthal angle, $\phi_{+}$, at the top substrate uniformly varies along the path, whereas the angle $\phi_{-}$is kept fixed. Then the MEP obtained at the initial step with $U=U_{0}$ is used as the initial approximation for the MEP computed at the voltage $U=U_{1}=U_{0}+\Delta U$. More generally, our approach can be described as the iteration procedure where the MEP computed at the step with $U=U_{i}$ serves as the starting approximation for the MEP evaluated at the subsequent step with the voltage $U_{i+1}=U_{i}+\Delta U$. Clearly, by using such a smooth continuation of the MEPs we assume that variations of the voltage result in continuously dependent on $U$ deformations of the MEPs.

Figure 4a shows the energies of director configurations along the MEPs computed at the anchoring energy strengths $W_{\phi}=0.05 \mathrm{~mJ} / \mathrm{m}^{2}$ and $W_{\theta}=0.5 \mathrm{~mJ} / \mathrm{m}^{2}$ when the voltage increases from $U=1.37 \mathrm{~V}$ to $U=1.38 \mathrm{~V}$. The curves for the energy reveal the presence of the pronounced peak that increases steeply as the voltage approaches $U=1.38 \mathrm{~V}$. Figure 5 demonstrates how out-ofplane deformations of the saddle-point structures develop in the course of the transition that might be called the director slippage-to-anchoring breaking transition. The profiles of the tilt angle for these structures are presented in Fig. 6.

An alternative initial approximation is the so-called anchoring breaking approximation used in Ref. [41. In this approximation the uniform twist from $\phi_{-}=0$ to $\phi_{+}$ is superimposed by the out-of-plane director deformation with the tilt angle $\theta$ varying from $\theta_{-}=0$ to $\theta_{+}$. In the first half of the path the tilt angle $\theta_{+}=0$ at the top substrate, increases from zero up to $\theta_{+} \approx \pi / 2$. Then in the second half of the path the angle $\theta_{+}$goes back to its initial value $\theta_{+}=0$ at the final state. So, the anchoring breaking initial guess for the MEP assumes that, for the transition state (saddle-point state), the CLC director 


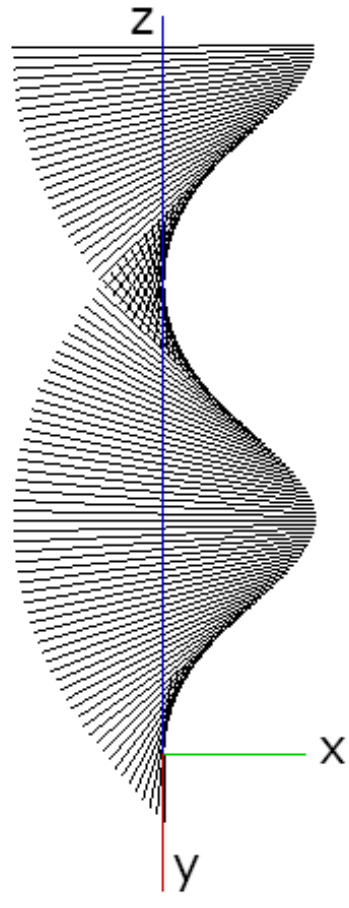

(a) $U=1.37 \mathrm{~V}$

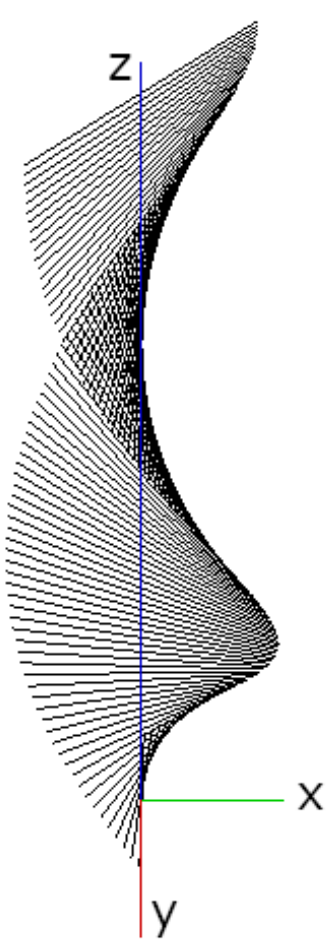

(b) $U=1.372 \mathrm{~V}$

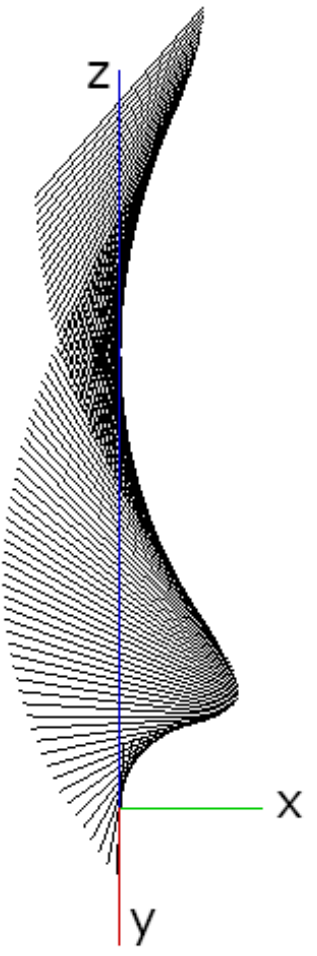

(c) $U=1.375$

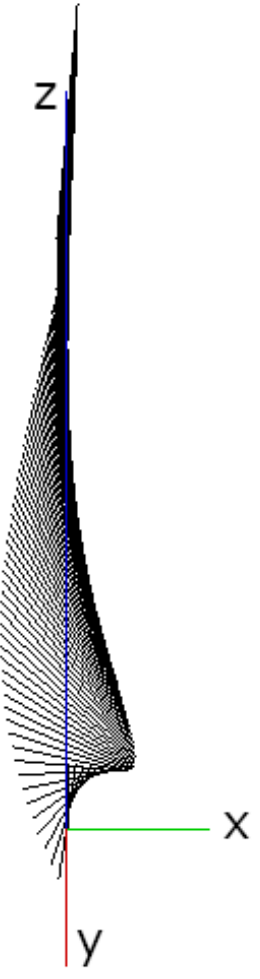

(d) $U=1.38 \mathrm{~V}$

Figure 5: Saddle-point structures for the MEPs with the energy curves depicted in Fig. 4a Parameters are listed in the caption of Fig. 3 .

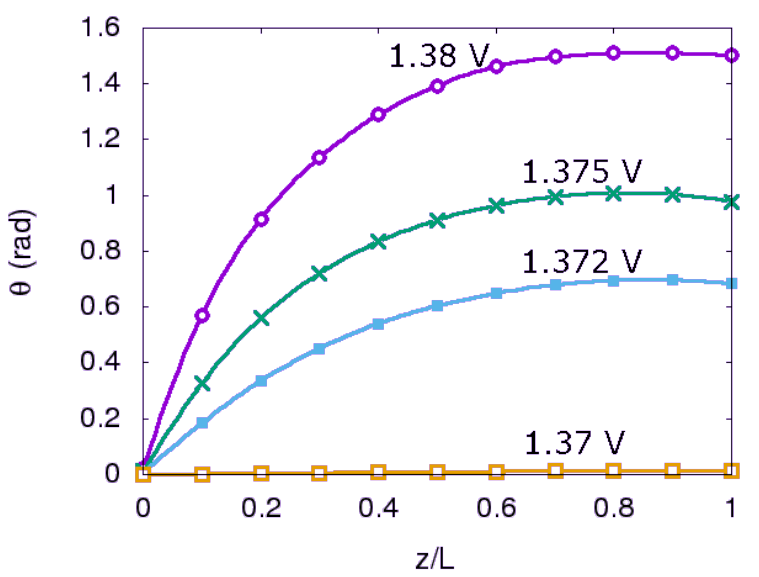

Figure 6: Profiles of the tilt angle for the saddle point structures presented in Fig. 5 .

at the top substrate is nearly normal to the bounding surface.

From the above discussion, at voltages higher than approximately $1.38 \mathrm{~V}$, the saddle point structure is characterized by pronounced tilt (out-of-plane) deformations. So, the high voltage regime, the director slippage and anchoring breaking approximations will produce identical results for the MEPs. These MEPs can now be smoothly continued to the region of low voltages. Figure $4 \mathrm{~b}$ presents the curves for the energies of director structures along the MEPs evaluated at when the voltage decreases from $U=1.122 \mathrm{~V}$ to $U=1.119 \mathrm{~V}$. It can be seen that behaviour of the peak corresponding to the energy of the saddle point structure bears a close resemblance to the director slippage-to-anchoring breaking transition that takes place at higher voltages (see Fig. 4a). The only difference is the direction of the voltage changes. So, the energy curves for the MEPs continued from high voltages to low ones show the behaviour that can be interpreted as the anchoring breaking-todirector slippage transition.

The energy barriers computed from a smooth continuation of the MEPs when an increase in applied voltage is followed by a decrease back to low voltages are depicted in Fig. 7. Clearly, the curves form the hysteresis loop. Figure 7 also demostrates what happen to the hysteresis loop when the polar anchoring strength is weakened. 


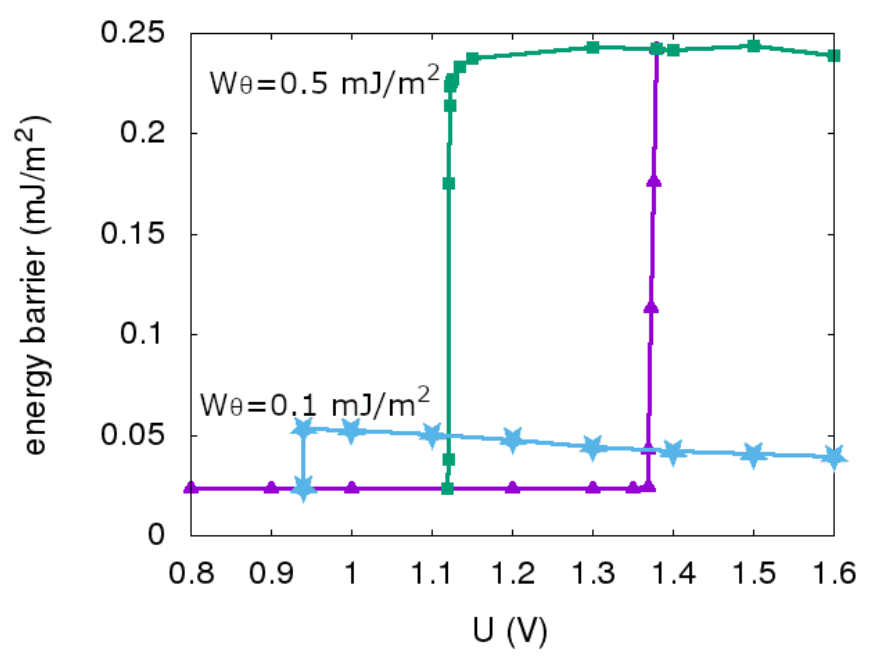

Figure 7: Hysteresis loop in voltage dependence of the energy barriers computed for smoothly continued MEPs assuming the director slippage-to-anchoring breaking scenario (purple filled triangles) and the anchoring breaking-to-director slippage scenario (green filled squares and blue stars) at different values of the polar anchoring energy strength. Azimuthal anchoring energy strength is $W_{\phi}=0.05 \mathrm{~mJ} / \mathrm{m}^{2}$.

\section{CONCLUSION}

In this paper, we have studied electric field dependence of the minimum energy paths (MEPs) for the transition between helical structures from different metastable branches in the chiral nematic liquid crystal cell. Such pathways connect the branches that are parametrized by the free twisting number and are labeled by halfturn numbers. We have examined local stability of the twisted states forming the branches and have deduced the analytical expressions describing dependence of the Fréedericksz thresholds on the free twisting number. Our analysis have shown that the stability conditions with respect to out-of-plane fluctuations impose additional restrictions on the values of the free twisting number of metastable helical states. Such restrictions may considerably affect mechanisms of the pitch transitions.

The energy of the saddle-point structure (transition state) of the paths gives the energy barrier separating the metastable states. Therefore, the MEP and its saddle points characterize the mechanism (scenario) of the transition. We have employed the geodesic nudged elastic band (GNEB) method as a computational procedure to evaluate the MEPs. This method requires an initial guess for the path and various starting approximations can generally produce different MEPs.

In our approach, we have evaluated two parts of the electric field dependence of the MEPs as smooth continuations of the starting paths in the low and high voltage regions, respectively. At low voltages, the initial path was obtained using the director slippage approximation. It turned out that, when the voltage increases, the energy of the saddle point structure steeply grows in the interval close to the critical voltage of the director slippage-toanchoring breaking transition, $U \equiv U_{c \uparrow} \approx 1.38 \mathrm{~V}$. This is the voltage where the nearly planar transition state becomes tilted and assumes noticeable out-of-plane director deformation. In other words, this implies that the director slippage scenario transforms into the anchoring breaking scenario.

Similarly, when the MEP is smoothly continued from the high voltage region dominated by the anchoring breaking mechanism to the region of low voltages the director slippage scenario comes into play when the voltage is smaller than its critical value $U_{c \downarrow} \approx 1.12 \mathrm{~V}$ associated with the anchoring breaking-to-director slippage transition. Since $U_{c \downarrow}<U_{c \uparrow}$, the voltage dependence of the energy barrier is characterized by the hysteresis loop shown in Fig. 7.

Interestingly, the critical field $U_{c \downarrow}$ is lower than the Fréedericksz thresholds of both the initial and final states of the MEPs, $U_{\mathrm{th}}^{(1)} \approx 1.3 \mathrm{~V}$ and $U_{\mathrm{th}}^{(2)} \approx 1.67 \mathrm{~V}$. It means that the anchoring breaking scenario with the tilted saddle point structure may take place even if the initial and the final states are both planar. Similar remark applies to the full-turn state with $k=2$ that remains undistorted at $U_{c \uparrow}<U_{\mathrm{th}}^{(2)}$.

The effect of polar anchoring on the form of the hysteresis loop is illustrated in Fig. 7. It is shown that, when the polar anchoring strength $W_{\theta}$ is reduced by five times, the magnitude of energy barriers suffer similar reduction. The effect responsible for such behaviour is that the dominating contribution to the energy of the saddle-point structure appears to be governed by its surface part.

Referring to Fig. 7], it can also be seen that the hysteresis loop widens when the polar anchoring weakens. Such widening can be attributed to the anchoring induced lowering of the Fréedericksz thresholds for the helical states.

\section{ACKNOWLEDGMENTS}

This work was supported by the Russian Science Foundation under grant 19-42-06302.
[1] P. G. de Gennes and J. Prost, The Physics of Liquid Crystals (Clarendon Press, Oxford, 1993) p. 596.

[2] P. Oswald and P. Pieranski, Nematic and Cholesteric Liquid Crystals: Concepts and Physical Properies Illustrated by Experiments, The Liquid Crystals Book Series (Taylor \& Francis Group, London, 2005) p. 618.

[3] D.-K. Yang and S.-T. Wu, Fundamentals of Liquid Crystal Devices, Series in Display Technology (Wiley, Chich- 
ester, 2006) p. 378.

[4] S. Obayya, M. F. O. Hameed, and N. F. Areed, Computational Liquid Crystal Photonics: Fundamentals, Modelling and Applications (Wiley, Chichester, 2016) p. 264.

[5] H. K. Bisoyi, T. J. Bunning, and Q. Li, Stimuli-driven control of the helical axis of self-organized soft helical superstructures, Advanced Materials 30, 1706512 (2018)

[6] R. S. Zola and Q. Li, Stimuli-directed helical axis switching in chiral liquid crystal nanostructures, in Functional Organic and Hybrid Nanostructured Materials (John Wiley \& Sons, Ltd, 2018) Chap. 8, pp. 307-357.

[7] A. Ryabchun and A. Bobrovsky, Cholesteric liquid crystal materials for tunable diffractive optics, Advanced Optical Materials 6, 1800335 (2018)

[8] R. Balamurugan and J.-H. Liu, A review of the fabrication of photonic band gap materials based on cholesteric liquid crystals, Reactive and Functional Polymers 105, 9 (2016)

[9] V. Vinogradov, A. Khizhnyak, L. Kutulya, Y. Reznikov, and V. Reshetnyak, Photoinduced change of cholesteric LC-pitch, Mol. Cryst. Liq. Cryst. 192, 273 (1990).

[10] R. A. v. Delden, T. Mecca, C. Rosini, and B. L. Feringa, A chiroptical molecular switch with distinct chiral and photochromic entities and its application in optical switching of a cholesteric liquid crystal, Chemistry - A European Journal 10, 61 (2004).

[11] R. Eelkema, Photo-responsive doped cholesteric liquid crystals, Liq. Cryst. 38, 1641 (2011).

[12] N. Katsonis, E. Lacaze, and A. Ferrarini, Controlling chirality with helix inversion in cholesteric liquid crystals, $\mathrm{J}$. Mater. Chem. 22, 7088 (2012)

[13] T. N. Orlova, R. I. Iegorov, and A. D. Kiselev, Lightinduced pitch transitions in photosensitive cholesteric liquid crystals: Effects of anchoring energy, Phys. Rev. E 89, $012503(2014)$

[14] Z.-g. Zheng, L. Yannian, H. K. Bisoyi, L. Wang, T. J. Bunning, and Q. Li, Three-dimensional control of the helical axis of a chiral nematic liquid crystal by light, Nature 531, 352 (2016)

[15] H. K. Bisoyi and Q. Li, Light-directed dynamic chirality inversion in functional self-organized helical superstructures, Angewandte Chemie International Edition 55, 2994 (2016)

[16] H. Huang, T. Orlova, B. Matt, and N. Katsonis, Longlived supramolecular helices promoted by fluorinated photoswitches, Macromolecular Rapid Communications 39, 1700387 (2018).

[17] S. Kurihara, T. Kanda, T. Nagase, and T. Nonaka, Photochemical color switching behavior of induced cholesteric liquid crystals for polarizer free liquid crystalline devices, Appl. Phys. Lett. 73, 2081 (1998).

[18] T. J. White, A. S. Freer, N. V. Tabiryan, and T. J. Bunning, Photoinduced broadening of cholesteric liquid crystal reflectors, J. Appl. Phys. 107, 073110 (2010).

[19] T. Kosa, L. Sukhomlinova, L. Su, B. Taheri, T. J. White, and T. J. Bunning, Light-induced liquid crystallinity, Nature 485, 347 (2012).

[20] J. P. Vernon, A. D. Zhao, R. Vergara, H. Song, V. P. Tondiglia, T. J. White, N. V. Tabiryan, and T. J. Bunning, Photostimulated control of laser transmission through photoresponsive cholesteric liquid crystals, Opt. Express 21, 1645 (2013).

[21] R. D. Kamien and J. V. Selinger, Order and frustration in chiral liquid crystals, Journal of Physics: Condensed
Matter 13, R1 (2000)

[22] N. J. Mottram and S. J. Hogan, Multiple solutions in twisted nematic liquid crystal layers, Continuum Mech. Thermodyn. 9, 213 (1997).

[23] V. A. Belyakov and E. I. Kats, Surface coupling and temperature variations of pitch in thin cholesteric layers, JETP 91, 488 (2000).

[24] V. A. Belyakov, P. Oswald, and E. I. Kats, Temperature pitch variations in planar cholesteric layers: The role of fluctuations and surface anchoring, JETP 96, 915 (2003).

[25] S. P. Palto, On mechanisms of spiral pitch variations in thin cholestric layers confined between two surfaces, JETP 121, 308 (2002), (in Russian).

[26] A. D. Kiselev and T. J. Sluckin, Twist of cholesteric liquid crystals cells: Stability of helical structures and anchoring energy effects, Phys. Rev. E 71, 031704 (2005)

[27] G. McKay, Bistable surface anchoring and hysteresis of pitch jumps in a planar cholesteric liquid crystal, Eur. Phys. J. E 35, 74 (2012).

[28] I. Lelidis, G. Barbero, and A. L. Alexe-Ionescu, Cholesteric pitch transitions induced by mechanical strain, Phys. Rev. E 87, 022503 (2013)

[29] G. Barbero, W. Zheng, and B. Zappone, Twist transitions and force generation in cholesteric liquid crystal films, Journal of Molecular Liquids 267, 242 (2018), special Issue Dedicated to the Memory of Professor Y. Reznikov.

[30] H. Zink and V. A. Belyakov, Temperature hysteresis of the change in the cholesteric pitch and surface anchoring in thin planar layers, JETP 85, 285 (1997).

[31] J. V. Gandhi, X.-D. Mi, and D.-K. Yang, Effect of surface alignment layers on the configurational transitions in cholesteric liquid crystals, Phys. Rev. E 57, 6761 (1998).

[32] H. Zink and V. A. Belyakov, Studies of the temperature pitch variations in planar cholesteric layer and surface anchoring, Mol. Cryst. Liq. Cryst. 329, 1069 (1999).

[33] H. G. Yoon, N. W. Roberts, and H. F. Gleeson, An experimental investigation of discrete changes in pitch in a thin, planar chiral nematic device, Liq. Cryst. 33, 503 (2006).

[34] P. F. Bessarab, V. M. Uzdin, and H. Jónsson, Method for finding mechanism and activation energy of magnetic transitions, applied to skyrmion and antivortex annihilation, Computer Physics Communications 196, 335 (2015).

[35] P. Hänggi, P. Talkner, and M. Borkovec, Reaction-rate theory: fifty years after Kramers, Rev. Mod. Phys. 62, $251(1990)$

[36] W. T. Coffey, D. A. Garanin, and D. J. Mccarthy, Crossover formulas in the Kramers theory of thermally activated escape rates - Application to spin systems, in Advances in Chemical Physics (John Wiley \& Sons, Ltd, 2007) Chap. 5, pp. 483-765.

[37] P. Goldbart and P. Ao, Intrinsic torsional viscosity of nematic liquid crystals, Phys. Rev. Lett. 64, 910 (1990)

[38] P. M. Goldbart and P. Ao, Intrinsic torsional viscosity in a narrow tube of nematic liquid crystal, Molecular Crystals and Liquid Crystals 198, 455 (1991)

[39] H. Kusumaatmaja and A. Majumdar, Free energy pathways of a multistable liquid crystal device, Soft Matter 11, 4809 (2015).

[40] A. V. Ivanov, P. F. Bessarab, E. V. Aksenova, V. P. Romanov, and V. M. Uzdin, Energy surface and minimum energy paths for Fréedericksz transitions in bistable 
cholesteric liquid crystals, Phys. Rev. E 93, 042708 (2016).

[41] S. S. Tenishchev, A. D. Kiselev, A. V. Ivanov, and V. M. Uzdin, Multiple minimum-energy paths and scenarios of unwinding transitions in chiral nematic liquid crystals, Phys. Rev. E 100, 062704 (2019).

[42] R. B. Meyer, Effects of electric and magnetic fields on the structure of cholesteric liquid crystals, Applied Physics Letters 12, 281 (1968)

[43] D. W. Berreman and W. R. Heffner, New bistable liquidcrystal twist cell, J. Appl. Phys. 52, 3032 (1981).

[44] M. E. Becker, J. Nehring, and T. J. Scheffer, Theory of twisted nematic layers with weak boundary coupling, Journal of Applied Physics 57, 4539 (1985).

[45] P. Schiller, Equilibrium structures of planar nematic and cholesteric films in electric fields, Phase Transitions 29, 59 (1990)

[46] H. Hirning, W. Funk, H.-R. Trebin, M. Schmidt, and H. Schmiedel, Threshold behavior and electro-optical properties of twisted nematics layers with weak anchoring in the tilt and twist angle, J. Appl. Phys. 70, 4211 (1991).

[47] I. I. Smalyukh, B. I. Senyuk, P. Palffy-Muhoray, O. D.
Lavrentovich, H. Huang, E. C. Gartland, V. H. Bodnar, T. Kosa, and B. Taheri, Electric-field-induced nematic-cholesteric transition and three-dimensional director structures in homeotropic cells, Phys. Rev. E 72, $061707(2005)$

[48] S. S. Choi, S. M. Morris, W. T. S. Huck, and H. J. Coles, Electrically tuneable liquid crystal photonic bandgaps, Advanced Materials 21, 3915 (2009)

[49] A. Y. Val'kov, E. V. Aksenova, and V. P. Romanov, First-order and continuous Fréedericksz transitions in cholesteric liquid crystals, Phys. Rev. E 87, 022508 (2013).

[50] A. Rapini and M. Papoular, Distortion d'une lamelle nematique sous champ magnetique. Conditions d'ancrage aux parois, J. Phys. (Paris) Colloq. C4 30, 54 (1969).

[51] A. Bogi and S. Faetti, Elastic, dielectric and optical constants of 4'-pentyl-4-cyanobiphenyl, Liquid Crystals 28, 729 (2001)

[52] H. Jónsson, G. Mills, and K. Jacobsen, Nudged elastic band method for finding minimum energy paths of transitions, B.J. Berne, G. Ciccotti, D.F. Coker (Eds.), Classical and Quantum Dynamics in Condensed Phase Simulations, World Scientific (1998), 385 (1998). 